\title{
APPLICATION OF COORDINATE EQUIPMENT FOR MEASURING PARAMETERS OF GEAR
}

\author{
Julia Skovorodkina \\ National Scientific Centre "Institute of Metrology", Kharkov, Ukraine \\ +38 05770497 85, julia.skovorodkina@metrology.kharkov.ua
}

\section{Annotation}

The report's goal is minimizing of uncertainty type $A$ while gear measuring on the coordinate measuring machine. Providing the necessary accuracy of the new gear measurements. The improvement necessity of unit's transmission from coordinate - measuring machine to specialized gear measuring devices method. The results of our study can be used to develop a protocol of international comparisons.

For solution the problem to achieve the required accuracy of measurements of the original product in Ukraine it is necessary to determine for which subordinate measuring instruments and products it is finally required. For this purpose, there should be analyzed the requirements for accuracy of manufacture of gears and, in particular, the requirements for the parameters of gear wheels that define the accuracy and smoothness of the mechanisms where gears are used. These parameters are:

- single pitch deviation;

- cumulative pitch deviation;

- profile total deviation;

- helix total deviation.

Currently, regulations governing the tolerances for the gears on the mentioned parameters, are the ISO 1328-1, which harmonize the requirements for the production of gears with the requirements of European standards and the GOST 1643 in accordance to which requirements for tolerances for the manufacture of gears in Ukraine were specified for the last 50 years $[1,2]$.

Comparative data of requirements for tolerances, for profile parameters, for the most accurate gears (3.4 degrees of accuracy), and also for the most used in machine-tool building, machine building (5-7 degrees of accuracy) are shown in Fig. 1

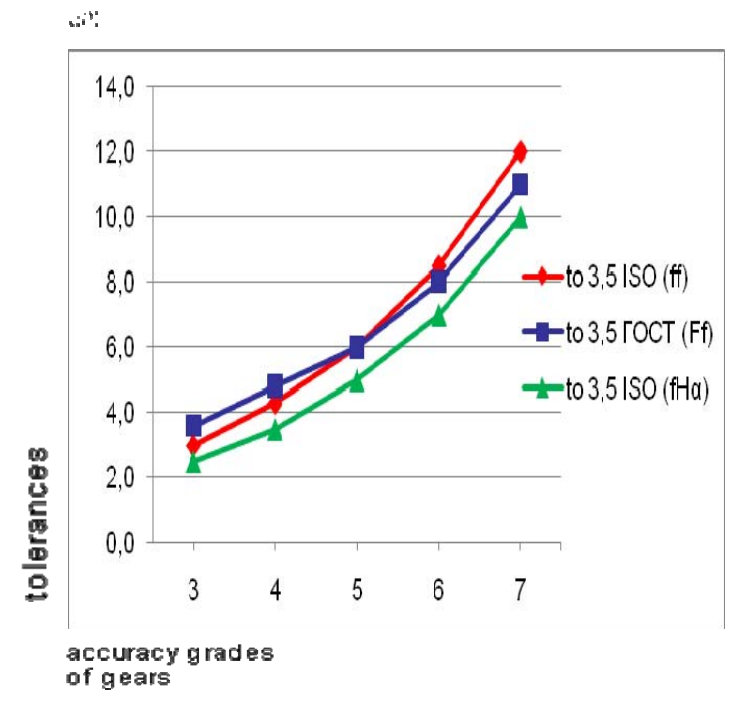

a)

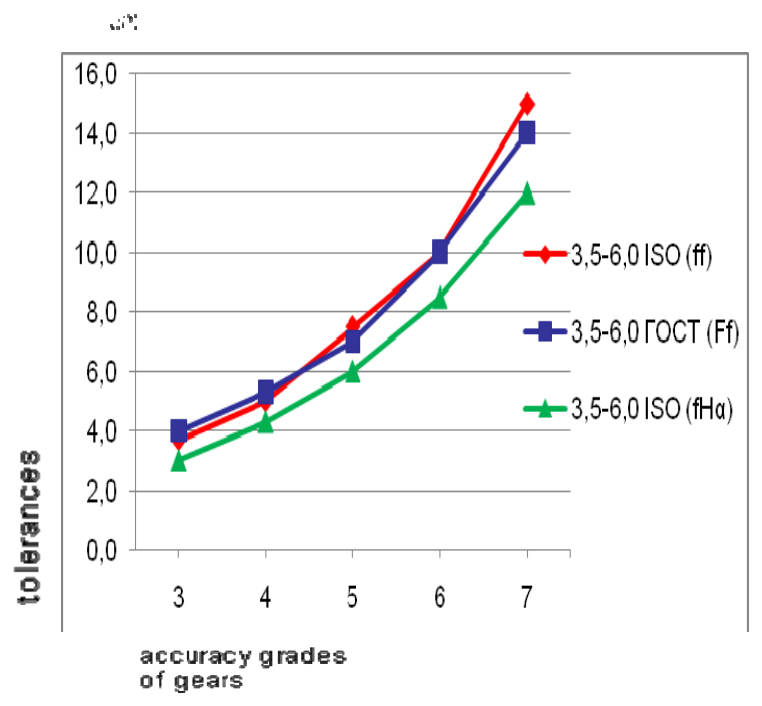

b) 


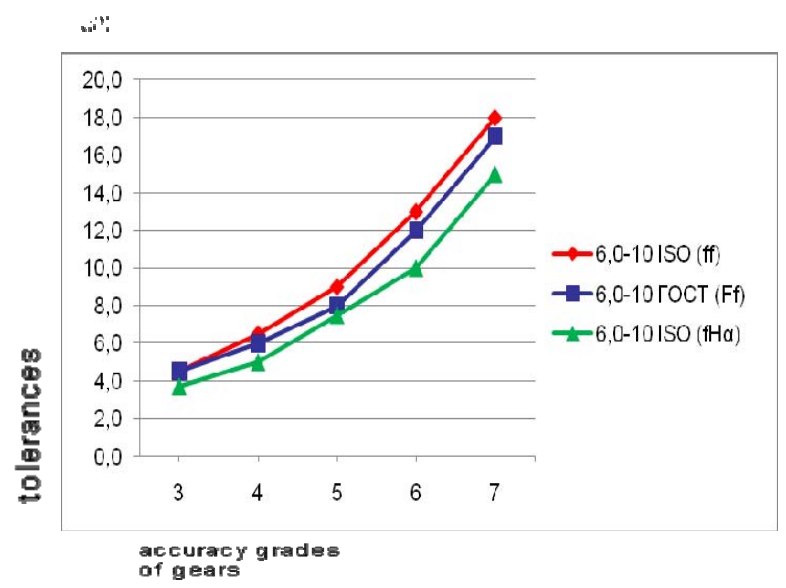

c)

Fig.1 (a, b, c). Requirements for the tolerances on the helix form deviation ( $f f, f H \alpha)$ for pitch diameter up to $125 \mathrm{~mm}$ and up to 3.5 units; 3.5-6.0; 6.0-10.0 $\mu \mathrm{m}$ in accordance of GOST 1643 and ISO 1328.

As the analysis of graphs for profile parameters shows (taking into account that the data on tolerances for deviations in the profile in accordance with GOST 1643 is normalized only by one quantity that is the total manifestation of $f_{f}$ and $f_{H \alpha}$ ), the requirements of GOST 1643 and ISO 1328 practically coincide for high-precision degrees, and for less accurate degrees of GOST 1643 sets more stringent demands for the accuracy of manufacturing gears than ISO 1328. Thus, the current system for ensuring the uniformity of measurements in the field of parameters of gears in Ukraine can be used further to provide the necessary accuracy of manufacturing gears. This system is based on - the state primary standard of unit length for the parameters of the involute surfaces and the angle of inclination of the tooth line.

Further improvement of the standard with the use of CMM allows to expand the possibilities of measuring other types of gears and their other parameters.

The first international comparisons of the measurement of gear parameters using CMM were carried out in 2008-2010, where the coordinating country was Germany (RTV) [3], Ukraine (NSC "Institute of Metrology") also participated in these comparisons among the other 6 countries: Germany, USA, Japan, China, Thailand, Great Britain.

Currently, Ukraine, along with Belarus and Russia, participates in additional international comparisons using CIM within COOMET, the coordinating country is Germany. Comparisons are at the final stage.

Measures provided by the NSC "Institute of Metrology", Ukraine: cogwheel, angle of inclination of the tooth line, involute measure were used as measures of comparison.

In prospect, it is proposed to participate in the research of improving the scientific and technical capabilities in coordinate metrology.

The main goal is to provide industrial requirements and improve the capabilities of scientific institutions using coordinate technology.

To achieve these goals, it is proposed:

- to develop and implement modern methods of error accounting and their elimination to obtain a submicron level of uncertainty while using of CMM;

- to develop methods for selecting of a measurement strategy and traceability paths.

In carrying out these works it is planned to make comparisons, both bilateral and multilateral, to evaluate the method - the development of methods.

With participation in these international works it is intended to use our results while measuring the parameters of the gears on the CMM.

Returning to the coordinate measuring equipment belonging to the NSC "Institute of Metrology", we can say the following:

According to the manufacturer's passport data, the limit of the permissible error of the NSC CMM is: 
where $L$ - in meters

$$
\Delta=\left(1,40+\frac{L}{300}\right), \mu \mathrm{m},
$$

In accordance with the "Guide to the expression of uncertainty" [3], from the above expression, a standard uncertainty of type $B$ can be determined:

$$
\left.\mathrm{U}_{\mathrm{B}}=\frac{\Delta}{k \sqrt{3}}\right),
$$

Where $k$ - is a coefficient that takes into account the effect of correlation at $P=0.95$.

For the maximum radius of the main circle of the involute measures, the type $B$ uncertainty is $0.60 \mu \mathrm{m}$.

The structure of type B uncertainty includes all inaccuracies admitted by the program of certification of CMM, in particular:

- nonsphericity of the tip; machines;

- the degree of compensation for the inaccuracy in the movement of the guide

- the degree of compensation for the inaccuracy of the displacement of the tip, etc.

These values can not be changed during the application of CMM and therefore the main influencing factor, from the point of view of CMM users, is a reduction in the random component of type A uncertainty.

Based on these provisions, we can assume that to obtain an expanded uncertainty of not more than $1.2 \mu \mathrm{m}$, it is necessary to reduce the random component (type A uncertainty) to a minimum to ensure reliable transmission of accuracy between the standards

Analysis of the results of the experimental observations of the NSC "Institute of Metrology" has determined the main areas of research that must be done to improve the accuracy of measuring on the parameters of the gears on the coordinate measuring machine.

An important factor that affects the measurement results is the speed of the touch probe during the measurement. The table shows the RMS of results of measuring the accumulated error of the pitch of the gear wheel under the same external conditions and with the same setting on the measuring position, at a constant speed of the probe movement and at several different speeds.

The results of measurements of the accumulated error in the pitch of the gear

\begin{tabular}{|l|c|c|}
\hline \multicolumn{1}{|c|}{ Parameters of gears } & $\begin{array}{c}\text { RMSD of repeated } \\
\text { measurements, } \\
V=\text { const }\end{array}$ & $\begin{array}{c}\text { RMSD of repeated } \\
\text { measurements, } V=v a r\end{array}$ \\
\hline $\begin{array}{l}\text { Cumulative pitch deviation on } \\
\text { the right profile, } \mu \mathrm{m}\end{array}$ & 0,090 & 0,220 \\
\hline $\begin{array}{l}\text { Cumulative pitch deviation the } \\
\text { left profile, } \mu \mathrm{m}\end{array}$ & 0,093 & 0,170 \\
\hline
\end{tabular}

As the results of experimental studies show, the standard deviation of the results of measurements at a constant speed is almost 2 times less than at with a varying speed.

The optimum speed can be determined from the comparison of measurement results obtained during international comparisons both within the framework of EURAMET and COOMET.

In addition to the speed factor of the probe movement while measuring the angle of inclination of the tooth line, in order to obtain unambiguous results, measurements should be made in the forward and inverted position of the measure vertically. The deviation of the axis in the forward and inverted position of the measure should not exceed $1 \mu \mathrm{m}$. In this case, the movement of the tip should be in one direction. For the result of the measurement, the arithmetic mean of the results obtained should be taken.

When measuring the pitch of the gear wheel and the accumulated step error, it is possible to move the probe without rebuilding the entire measuring head. In Fig. 2 is shown two schemes for measuring the pitch of the gear fixed to the measuring position. 


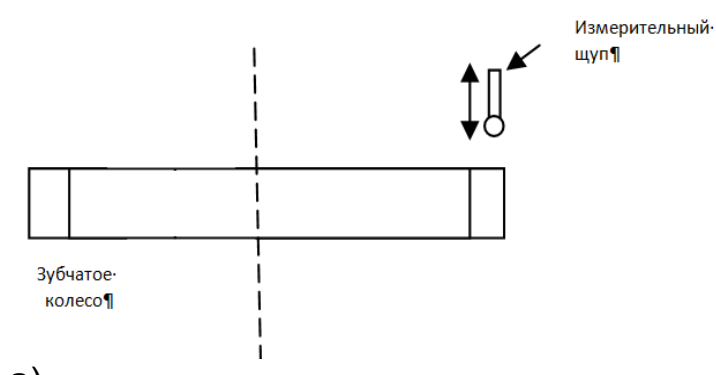

a)

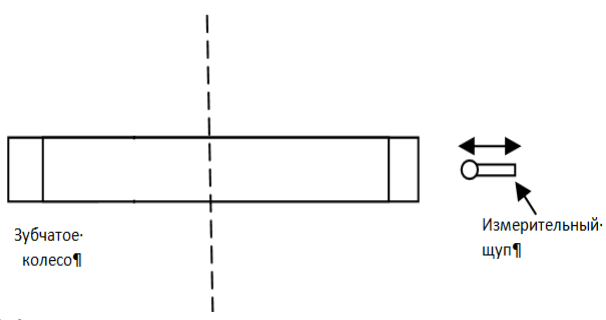

b)

Fig. 2. The deviation of the pitch of the gear fixed to the measuring position

Variant a) assumes carrying out of measurements without adjustment of a measuring head at an option b) assumes a minimum of three changes of a measuring head.

Since the measurement of gears refers to the type of geometric measurements, a significant effect on their result is maintained by the required temperature. In the future, it is necessary to determine the optimal location of the temperature sensors, their number depending on the type of measures measured at the CMM.

As shown by our experiments, the temperature deviation should not exceed $(20 \pm 0.3)^{\circ} \mathrm{C}$ to maintain the required accuracy.

It is also necessary to research the method of transferring a unit from the state standard, into which CMM will be included, to existing special measuring instruments that are verified (calibrated) by means of measures of the parameters of the gears.

Taking into account the above, it is possible to determine the main directions for carrying out further research in order to achieve the required accuracy of measurements of measures of the parameters of gears on a coordinate measuring machine:

- measurement in one position of the CMM measuring head;

- strictly prescribe the position of the measure in the measurement process.

- Determine the algorithm and the measurement mode.

The use of coordinate technology in measuring the parameters of the gears makes it possible to measure new sizes and parameters of the gears with the required accuracy.

It requires improvement of methods of transferring a unit from coordinate-measuring equipment to specialized instruments.

Also the results of this work will be used as a basis for the normative documentation on measurement methods for measuring the parameters of the gears.

\section{Bibliography}

1. GOST 1643-81 cylindrical gear wheels. Tolerances

2. ISO 1328-1: 2006 Wheels cylindrical. ISO Accuracy System Part 1. Terms and definitions of concepts and the establishment of tolerance tolerances of the lateral surfaces of the gears. Wheels cylindrical. Tolerances.

3. Guidelines for the expression of uncertainty RMG 43-2001.

4. EUROMET Supplementary Comparison EURRAMET.L-S24. Involute Gear Standards. Final Report. October 2013 